\title{
RND type efflux pump system MexAB-OprM of pseudomonas aeruginosa selects bacterial languages, 3-oxo-acyl-homoserine lactones, for cell-to-cell communication
}

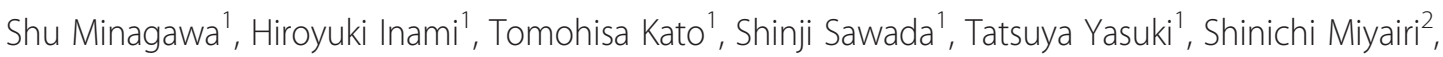
Manabu Horikawa ${ }^{3}$, Jun Okuda ${ }^{1}$ and Naomasa Gotoh ${ }^{1 *}$

\begin{abstract}
Background: Bacteria release a wide variety of small molecules including cell-to-cell signaling compounds. Gramnegative bacteria use a variety of self-produced autoinducers such as acylated homoserine lactones (acyl-HSLs) as signal compounds for quorum sensing (QS) within and between bacterial species. QS plays a significant role in the pathogenesis of infectious diseases and in beneficial symbiosis by responding to acyl-HSLs in Pseudomonas aeruginosa. It is considered that the selection of bacterial languages is necessary to regulate gene expression and thus it leads to the regulation of virulence and provides a growth advantage in several environments. In this study, we hypothesized that RND-type efflux pump system MexAB-OprM of $P$. aeruginosa might function in the selection of acyl-HSLs, and we provide evidence to support this hypothesis.

Results: Loss of MexAB-OprM due to deletion of mexB caused increases in QS responses, as shown by the expression of gfp located downstream of the lasB promoter and LasB elastase activity, which is regulated by a LasR3-oxo-C12-HSL complex. Either complementation with a plasmid containing wild-type mexB or the addition of a LasR-specific inhibitor, patulin, repressed these high responses to 3-oxo-acyl-HSLs. Furthermore, it was shown that the acyl-HSLs-dependent response of $P$. aeruginosa was affected by the inhibition of MexB transport activity and the mexB mutant. The P. aeruginosa MexAB-OprM deletion mutant showed a strong QS response to 3-Oxo-C10-HSL produced by Vibrio anguillarum in a bacterial cross-talk experiment.

Conclusion: This work demonstrated that MexAB-OprM does not control the binding of LasR to 3-oxo-Cn-HSLs but rather accessibility of non-cognate acyl-HSLs to LasR in P. aeruginosa. MexAB-OprM not only influences multidrug resistance, but also selects acyl-HSLS and regulates QS in P. aeruginosa. The results demonstrate a new QS regulation mechanism via the efflux system MexAB-OprM in $P$. aeruginosa.
\end{abstract}

\section{Background}

Gram-negative bacteria use a variety of self-produced autoinducers such as acylated homoserine lactones as a language for quorum sensing (QS) within and between bacterial species. Several bacterial species synthesize specific acylated homoserine lactones (acyl-HSLs) by means of a LuxI-type enzyme, and respond to cognate acylHSL by using a LuxR-type intracellular receptor [1,2]. It

\footnotetext{
* Correspondence: ngotoh@mb.kyoto-phu.ac.jp

1 Department of Microbiology and Infection Control Science, Kyoto

Pharmaceutical University, Yamashina, Kyoto 607-8414, Japan

Full list of author information is available at the end of the article
}

is considered that the selection of bacterial languages is necessary to regulate gene expression and thus it leads to a growth advantage in several environments.

The opportunistic bacterium $P$. aeruginosa is widespread in various environments and utilizes two acyl-HSL signaling molecules, $\mathrm{N}$-(3-oxododecanoyl)-L-homoserine lactone (3-oxo-C12-HSL), and N-butanoyl-L-homoserine lactone (C4-HSL), and two receptor proteins, LasR and RhlR, respectively [3]. 3-oxo-C12-HSL binds to LasR and activates LasR function. The 3-oxo-C12-HSL-LasR complex regulates many genes, including the $r h l$ system [4-6]. Furthermore, P. aeruginosa uses a third signal,

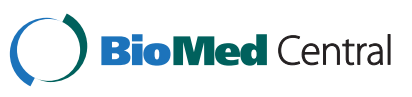


Pseudomonas quinolone signal (PQS) and the PqsR receptor protein [7]. Expression of many virulence factors is regulated by QS in $P$. aeruginosa [4-6,8,9]. Accordingly, a specific response to an autoinducer is important to determine the virulence of $P$. aeruginosa.

Analysis of the crystal structures of the N-terminal half of the $P$. aeruginosa full-length LasR or the crystal structure of $A$. tumefaciences full-length TraR, which is a homolog of $P$. aeruginosa LasR, in a complex with its cognate autoinducer has been performed $[6,10]$. These structural analyses indicated that the N-terminal half of the full length LuxR-type protein includes the dimerization domain and the acyl-HSL binding domain $[6,10]$. These reports indicated that the ligand binds to the N-terminal half of the full-length LuxR-type protein at an enclosed cavity far from the N-terminal dimerization region. It has been suggested that the acyl side-chain length of acyl-HSLs is not the main factor that determines the specificity of receptor protein binding $[6,10]$. It is considered that the binding model for the acyl-HSL-LuxR transcriptional protein family is common among Gram-negative bacteria [6,10]. However, it was shown that the responses to acyl-HSLs in $P$. aeruginosa are specific $[4,11]$. We hypothesize that there is an unidentified signal selection mechanism for the selection of acyl-HSLs according to the binding affinity of LasR in $P$. aeruginosa.

Resistance-nodulation-division (RND)-type efflux pumps are one type of antibiotic efflux system. RND-type efflux pumps are commonly found in gram-negative bacteria. RND family transporters catalyze the active efflux of many antibiotics and chemotherapeutic agents. They consist of an inner-membrane component belonging to the RND superfamily of secondary transporters, a channelforming outer membrane factor (OMF), and a periplasmic membrane fusion protein (MFP) to achieve the direct extrusion of substrates across the two membranes of gramnegative bacteria [12].

The major $P$. aeruginosa RND-type efflux pump, MexAB-OprM provides the bacterium natural resistance to a broad spectrum of antibiotics and is not just for antimicrobial resistance [12]. On the other hand, it was reported that MexAB-OprM participates in the efflux of acyl-HSLs from $P$. aeruginosa [13,14]. These reports indicated that $P$. aeruginosa cells are not freely permeable to 3-oxo-C12-HSL in contrast to C4-HSL. Instead, it was shown that MexAB-OprM is involved in the active efflux of 3-oxo-C12-HSL [13,14]. Furthermore, a MexAB-OprM deletion mutant has a decreased capacity to invade or transmigrate across MDCK cells [15]. It was considered that QS-regulated virulence factors are affected by the MexAB-OprM efflux pump activity.

In this study, we hypothesized that MexAB-OprM of $P$. aeruginosa might function in the selection of acyl-
HSLs, and we provide evidence to support this hypothesis. To examine the QS responses to several exogenous acyl-HSLs in $P$. aeruginosa, herein we focused on the las system because this system controls the $r h l$ system and the PQS system hierarchically in $P$. aeruginosa [2,5,7]. These studies indicate that MexAB-OprM prevents the access of exogenous 3-oxo-acyl-HSLs to LasR, and thus LasR binds specifically to 3-oxo-C12-HSL. The results demonstrate a new $\mathrm{QS}$ regulation mechanism via the efflux system MexAB-OprM in P. aeruginosa.

\section{Results}

\section{MexAB-OprM selects acyl-HSLs and regulates quorum sensing}

To determine whether or not the expression of the QS regulatory pathway in $P$. aeruginosa is influenced by exogenous acyl-HSLs substituted with 3-oxo-acyl groups with carbon numbers of 6 to 14, las $B$ transcription was measured by using a las $B$ promoter- $g f p$ reporter system. As a result, las $B$ transcription was most strongly induced by 3-oxo-C12-HSL, which is a cognate acylHSL in $P$. aeruginosa KG7403 (DlasI $\Delta$ rhlI plasB-gfp) (Figure 1a). Moreover, transcription of las $B$ resulted in a response to exogenous acyl-HSLs substituted with 3oxo-acyl-groups with 8-14 carbons. On the other hand, we analyzed the effect of C4-HSL on lasB expression. The results indicated that C4-HSL was not involved in las $B$ expression (data not shown). It was previously shown that C4-HSL did not affect LasR activation [5]. Our data agree with results in this report. These results indicate that regulation of QS in $P$. aeruginosa is affected by 3-oxo-Cn-HSLs besides 3-oxo-C12-HSL.

To determine whether or not the QS system in $P$. aeruginosa is regulated by MexAB-OprM, lasB transcription was measured by using KG7503 ( $\Delta$ lasI $\Delta$ rhlI $\Delta$ mexB plas $B-g f p)$. las $B$ transcription was induced to different levels by 3-oxo-Cn-HSLs with acyl chain lengths of C8 to 14 in KG7503, and compared to the results for the QS-negative mutant (Figure 1a). In this case, 3-oxo-C9HSL (5.2-fold) and 3-oxo-C10-HSL (2.8-fold) in particular were found to induce lasB expression. LasB elastase activity was measured by using a FRET-AGLA-based elastase assay, similar to the lasB-gfp reporter assay (Figure 1b). The results showed that LasB activity agreed with the las $B$ transcription results (Figure 1).

The results indicate that the responses to 3-oxo- $\mathrm{Cn}$ HSLs were affected by deletion of the MexAB-OprM efflux pump, and MexAB-OprM played a role in the efflux of 3-oxo-Cn-HSLs with acyl chain lengths of C8 to 14 including 3-oxo-C12-HSL. However, the QS response was more strongly induced by 3-oxo-C9-HSL or 3-oxoC10-HSL than by 3-oxo-C12-HSL in the MexAB-OprM deletion mutant. These results suggest that the rates of 3-oxo-C9-HSL and 3-oxo-C10-HSL uptake were higher 

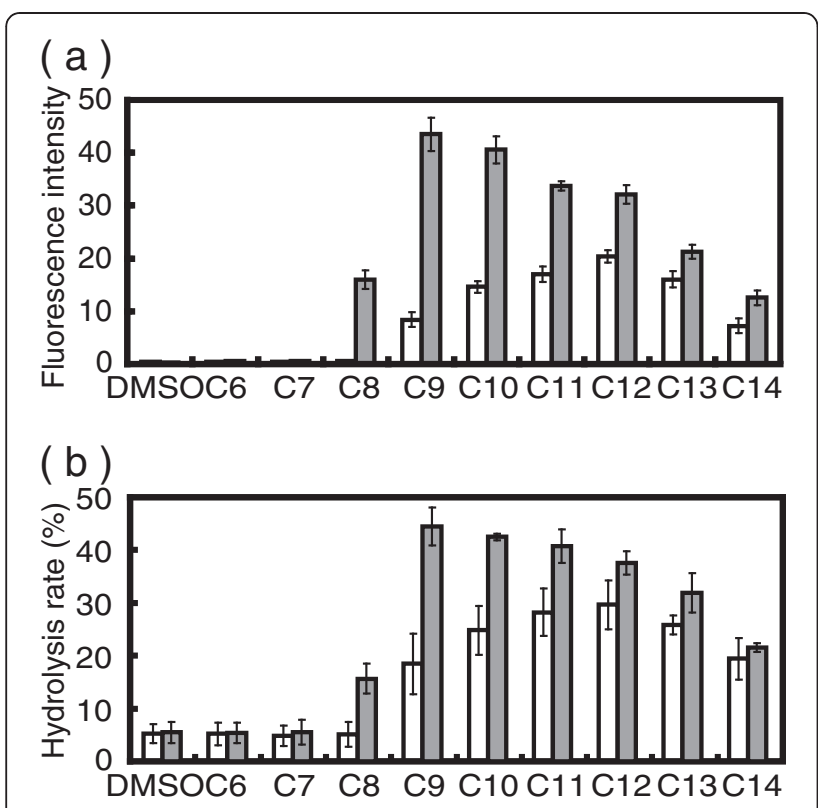

Figure 1 Acyl chain length of $\mathrm{N}$-(3-oxoacyl)-L-homoserine lactones has an effect on the regulation of las $B$ expression in the mexB deletion strain. (a) Individual cultures of KG7403 ( $\Delta$ /as) $\Delta$ rhll PlasB-gfp) and KG703 ( $\Delta$ lasl $\Delta$ rhll $\Delta$ mexB PlasB-gfp) were grown in $\mathrm{LB}$ medium containing $5 \mu \mathrm{M}$ 3-oxo-Cn-HSL, respectively. Transcription of las $B$ was determined by measurement of the fluorescence intensity (arbitrary units) depending on the amount of green-fluorescence protein (GFP) derived from PlasB-gfp (emission at $490 \mathrm{~nm}$; excitation at $510 \mathrm{~nm}$ ). (b) Individual culture supernatants of

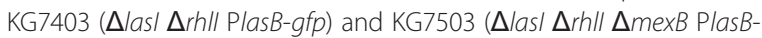
gfp) grown in LB medium containing $5 \mu \mathrm{M}$ 3-oxo-Cn-HSL, respectively, were assayed for LasB elastase activity. LasB activity was measured as the rate of hydrolysis of FRET-AGLA by the LasB protein. Hydrolysis rates were determined by measurement of fluorescence intensity depending on the $\mathrm{N}$-methylanthranilyl derivative derived from an elastase substrate; emission at $355 \mathrm{~nm}$ and excitation at $460 \mathrm{~nm}$. Open bars, KG7403; closed bars, KG7503. The data represent mean values of three independent experiments. Error bars represent the standard errors of the means. than that of 3-oxo-C12-HSL uptake, or that 3-oxo-C9HSL and 3-oxo-C10-HSL clearance rates may be lower than that of 3-oxo-C12-HSL. Alternatively, the binding affinities of 3-oxo-C9-HSL and 3-oxo-C10-HSL to LasR were stronger than that of 3-oxo-C12-HSL.

\section{MexAB-OprM plays a role in the efflux of 3-oxo-cn-HSLs in $P$. aeruginosa}

It is known that MexAB-OprM is expressed constitutively in wild-type $P$. aeruginosa, and MexAB-OprM exports a variety of substrates $[10,16]$. P. aeruginosa MexB has high sequence similarity $(69.8 \%$ amino acid identity and $83.2 \%$ similarity) with E. coli AcrB. The crystal structure of AcrB has been solved $[17,18]$. The efficiency of substrate binding most likely depends on the volume and the side-chain arrangements of the binding pocket $[17,18]$. We attempted to model the MexB three-dimensional structure using the crystal structure of AcrB from E. coli by S. Murakami et al. $[17,18]$. Phenylalanine residues in the pore domain and hydrophobic amino acid residues in the vestibule domain were assumed to play important roles in the transport of substrates. To analyze whether a mutation in the pore domain (Phe136Ala) and a mutation in the vestibule domain (Asp681Ala) of MexB are important for extrusion of substrates, the plasmid-borne mexB gene was mutagenized to obtain these single-amino-acid substitutions (Figure 2). Western immunoblotting subsequently confirmed that expression of wild-type and mutant MexBs was equivalent (data not shown). las $B$ transcription was more strongly induced by acyl-HSLs in the strain carrying the MexB Phe136Ala mutation compared to the strain carrying wild-type MexB. On the other hand, lasB expression in response to acyl-HSLs in the MexB Asp681Ala mutant was similar to the las $B$ expression pattern in the mex $B$ deletion mutant (Figure 2). las $B$ expression was affected by the mutation of these residues at positions 136 and 681 in MexB. These results indicate that MexB is necessary to extrude acyl-HSLs.

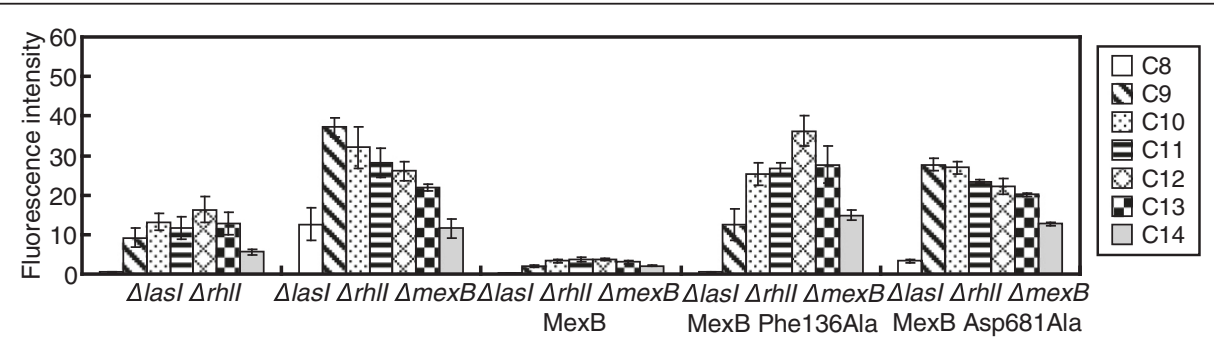

Figure 2 Mutation in the predicted porter domain of MexB affected the selective efflux of aycl-HSLs by MexAB-OprM. $P$. aeruginosa strains were grown in LB medium with acyl-HSLs, and lasB expression analyses were performed as described in Materials and Methods. Promoter activities are expressed in fluorescence intensities (arbitrary units) depending on amounts of green-fluorescence protein (GFP) derived from PlasBgfp at emission (490 nm; excitation, $510 \mathrm{~nm}$ ). The following MexB mutant strains were used: KG7403, KG7503, KG7503 carrying pKTA113 (wild-type MexB), pYT57 (MexB Phe136Ala), and pYT81 (MexB Asp681Ala). The data represent mean values of three independent experiments. Error bars represent the standard errors of the means. 
Furthermore, the responses to acyl-HSLs were analyzed in the presence of the MexAB-OprM specific inhibitor ABI (Figure 3). This analysis was carried out by using a lasB promoter-gfp reporter system with the $P$. aeruginosa cognate signal, 3-oxo-C12-HSL, and signals that strongly induce lasB expression, 3-oxo-C9-HSL and 3-oxo-C10-HSL. The results showed that the response to 3-oxo-C9-HSL or 3-oxo-C10-HSL was increased by $\mathrm{ABI}$ in a concentration-dependent manner in the MexAB-OprM activated strain (Figure $3 \mathrm{a}$ and b). However, the response to 3-oxo-C12-HSL was affected only by the addition of $0.5 \mu \mathrm{M}$ ABI (Figure 3c). The analysis of MexAB-OprM inhibition by ABI showed that the effect of $\mathrm{ABI}$ concentration on the response of 3-oxoC12-HSL was lower than that of 3-oxo-C9-HSL or 3oxo-C10-HSL (Figure 3). In contrast, the response was unaffected at a range of experimental concentrations of $\mathrm{ABI}$ in the QS-negative mexB deletion strain (Figure 3). These results indicate that MexAB-OprM extrudes 3oxo-Cn-HSLs from inside the cell, and that there are differences in the rates of efflux of 3-oxo-acyl-HSLs via MexAB-OprM.

The transcript levels of the mexB genes in the presence or absence of 3-oxo-C12-HSL were measured by semi-quantitative real-time reverse transcription-PCR (qRT-PCR). 3-oxo-C12-HSL had no effect on the mexB expression level in the QS-negative strain (data not shown), so MexAB-OprM is regulated through a QSindependent mechanism.

\section{Las $R$ is activated by accumulated intracellular noncognate acyl-HSLs}

It is known that the overexpressed QS regulator TraR responds to a variety of autoinducers in Agrobacterium tumefaciens $[10,19]$. Thus it appears that overexpressed regulatory proteins mis-respond to acyl-HSL signals. In the mexAB-oprM mutant, accumulated acyl-HSLs may be bound to LasR. To verify whether or not LasR responds to 3-oxo-Cn-HSLs (C8-C14) in the MexABOprM deletion mutant, transcription of las $B$ in response to 3-oxo-C9-HSL, 3-oxo-C10-HSL or 3-oxo-C12-HSL was analyzed by using the LasR inhibitor, patulin (Figure 4). lasB induction by 3-oxo-C9-HSL, 3-oxo-C10HSL or 3-oxo-C12-HSL decreased with or without MexAB-OprM in a patulin-concentration-dependent manner (Figure 4). These results indicate that 3-oxo- $\mathrm{Cn}$ HSLs were able to bind to LasR and modulate its activity. Furthermore, the lasB induction by 3-oxo-C9-HSL with the addition of $10 \mu \mathrm{M}$ patulin decreased to $10 \%$ of the level in the absence of patulin (Figure 4a). The addition of 3-oxo-C10-HSL or 3-oxo-C12-HSL with patulin decreased the lasB expression levels to $50 \%$ and $60 \%$, respectively (Figure $4 \mathrm{~b}$ and $\mathrm{c}$ ). These data indicate that the order of LasR-binding affinity for 3-oxo- $\mathrm{Cn}$ HSLs is: 3-oxo-C12-HSL > 3-oxo-C10-HSL > 3-oxo-C9HSL. These results suggest that acyl-HSL entry into the cell is likely to be passive and acyl-HSLs were extruded by MexAB-OprM. As a result of the accumulation of these acyl-HSLs in the MexAB-OprM mutant, a nonnatural response was induced.

\section{Selection of a bacterial language by MexAB-OprM in bacterial communication}

As we have shown here, $P$. aeruginosa responds to several 3-oxo-Cn-HSLs in vitro. However, it was not known whether this in vitro response to 3-oxo-Cn-HSLs was equivalent to a response to 3-oxo-Cn-HSLs in a natural environment. When grown in close proximity to the $P$. aeruginosa wild-type strain on LB plates, KG7004 ( $\Delta l a-$ $s I \Delta r h l I$ ) carrying pMQG003 (lasB promoter-gfp) exhibited bright-green fluorescence, but the $P$. aeruginosa reporter strain near the QS-negative strain, KG7004 ( $\Delta l a s I \Delta r h l I)$, did not show GFP fluorescence (Figure 5). These results clearly demonstrated that physiological concentrations of AHLs derived from PAO1 were de-

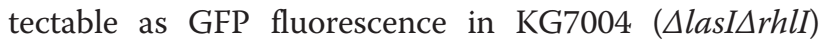

(a) 3-oxo-C9-HSL

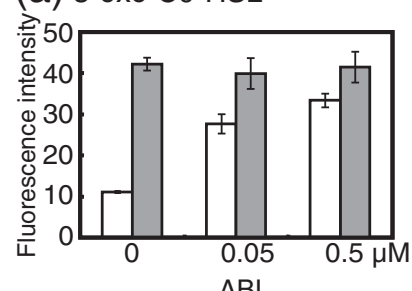

$\mathrm{ABI}$ (b) 3-oxo-C10-HSL

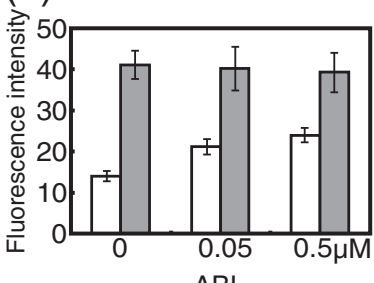

$\mathrm{ABI}$ (c) 3-oxo-C12-HSL

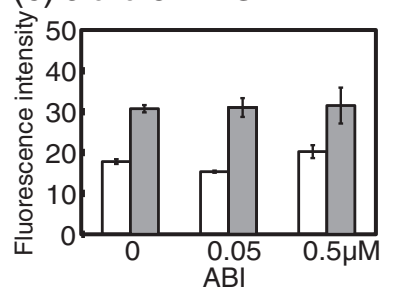

Figure 3 3-oxo-Cn-HSLs are selected by MexAB-OprM in $\boldsymbol{P}$. aeruginosa. Individual cultures of KG7403 ( $\Delta / a s / \Delta$ rh/l PlasB-gfp) and KG7503 ( $\Delta$ lasl $\Delta$ rhll $\Delta$ mexB PlasB-gfp) were grown in LB medium with $5 \mu \mathrm{M}$ 3-oxo-C9-HSL (a), 3-oxo-C10-HSL (b), or 3-oxo-C12-HSL (c), respectively. Transcription of las $B$ was determined by measurement of the fluorescence intensity (arbitrary units) depending on the amount of greenfluorescence protein (GFP) derived from PlasB-gfp; emission at $490 \mathrm{~nm}$ and excitation at $510 \mathrm{~nm}$. MexAB-OprM efflux activity was inhibited by 0 , 0.05 or $0.5 \mu \mathrm{M} \mathrm{ABI}$. Open bars, KG7403; closed bars, KG7503. The data represent mean values of three independent experiments. Error bars represent the standard errors of the means. 

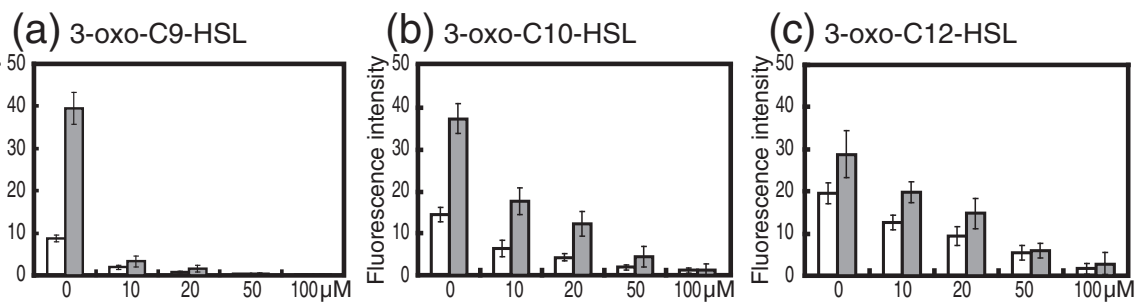

Figure 4 3-oxo-Cn-HSLs bind directly to LasR and the complexes are able to trigger lasB expression. Individual cultures of KG7403 ( $\Delta$ lasl $\Delta$ rhll PlasB-gfp) and KG7503 (Alasl $\Delta$ rhll $\Delta$ mexB PlasB-gfp) were grown in LB medium with 5 HM 3-oxo-C9-HSL (a), 3-oxo-C10-HSL (b), or 3-oxoC12-HSL (c) with $0,10,20,50$, or $100 \mu \mathrm{M}$ patulin, respectively. Transcription of las $B$ was determined by measuring the fluorescence intensity (arbitrary units) depending on the amounts of green-fluorescence protein (GFP) derived from PlasB-gfp; emission at $490 \mathrm{~nm}$ and excitation at $510 \mathrm{~nm}$. Open bars, KG7403; closed bars, KG7503. The data represent mean values of three independent experiments. Error bars represent the standard errors of the means.

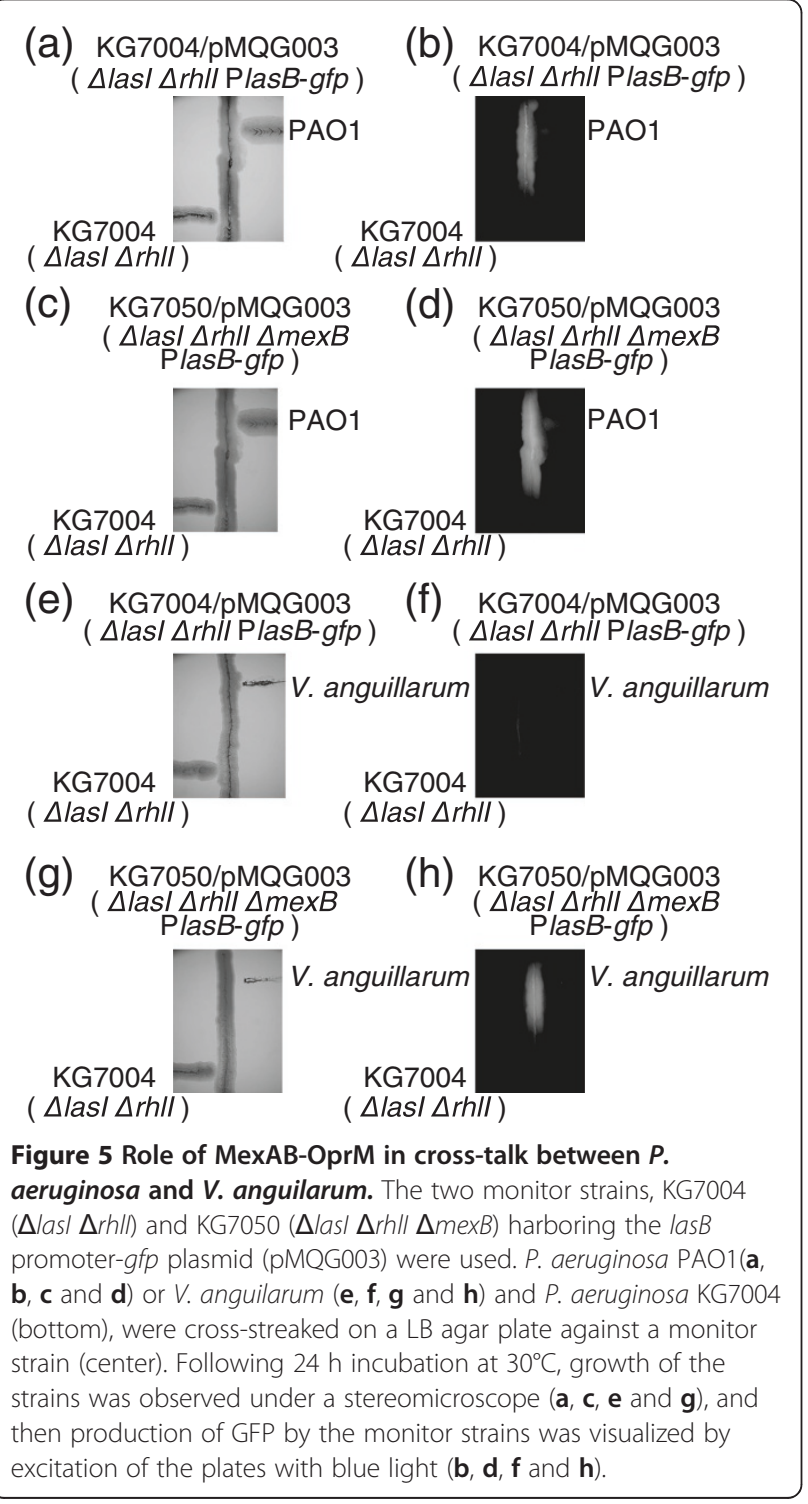

carrying pMQG003 (lasB promoter-gfp) (Figure 5). To examine the effect of MexAB-OprM on heterogeneous bacterial communication, $P$. aeruginosa was cocultivated with C. violaceum, P. chlororaphis, P. agglomerans, $P$. fluorescens or $V$. anguillarum (Figure 5 and Additional file 1: Figure S1). These bacteria are known to produce cognate acyl-HSLs [20-23]. It was shown that las $B$ expression by $P$. aeruginosa $\triangle m e x B$ was only strongly induced during co-cultivation with $V$. anguillarum (Figure 5 and Additional file 1: Figure S1). 3-oxoC10-HSL production by $V$. anguillarum was confirmed by TLC assays using Chromobacterium violaceum VIR07, in agreement with a previous report (Additional file 2: Figure S2) [22].

These results indicated cross-talk via 3-oxo-C10-HSL between $P$. aeruginosa and $V$. anguillarum with the $P$. aeruginosa mexAB-oprM deletion strain. The transport of acyl-HSLs by MexAB-OprM plays a role in regulation of cell-cell communication.

\section{Discussion}

The bacterial communication QS system plays many roles in the regulation of growth, biofilms, virulence and pathogenesis. Gram-negative bacteria produce specific acyl-HSLs, and then respond to specific signals. In $P$. aeruginosa, QS regulates many genes in response to the cognate 3-oxo-C12-HSL. The selection of cognate acylHSLs from among several autoinducers is a bacterial adaptation to environmental conditions. We showed that $P$. aeruginosa QS responds to exogenous acyl-HSLs substituted with 3-oxo-acyl-groups with between 8 and 14 carbons (Figure 1). P. aeruginosa LasR responds to a variety of AHLs with varying acyl chain lengths and activated LasR regulates the expression of many genes. An A. tumefaciens or C. violaceum QS reporter strain, which recognizes a broad range of acyl-HSLs, has been utilized to detect acyl-HSLs in many studies $[19,22,23]$. Based on these reports, it was suggested that TraR 
family proteins including LasR respond to several acylHSLs in un-natural conditions, in which the TraR family proteins are overexpressed.

The response to and specificity of the cognate bacterial language were analyzed in $P$. aeruginosa and $B$. cepacia [11]. These results suggest that bacteria have a selection mechanism for acyl-HSLs besides recognition of acylHSLs by the TraR family. In fact, LasR was activated by 3-oxo-C9-HSL or 3-oxo-C10-HSL in the same way as 3oxo-C12-HSL in the $P$. aeruginosa mexB deletion mutant (Figures. 1 and 2). Furthermore, the responses to acyl-HSLs were analyzed using a site-directed MexB mutant (Figure 2). These data indicated that lasB expression was affected by the substitutions Phe136Ala or Asp681Ala in MexB (Figure 2). In particular, the MexB Phe136Ala mutation affected the response to acyl-HSLs similar to that of the mexB deletion mutant (Figure 2). This result suggested that Phe136 in MexB played an important role in substrate extrusion by MexB. On the other hand, lasB expression increased in the MexB Asp681Ala mutant compared with wild-type MexB. This result suggested that the MexBAsp681Ala mutation induced the extrusion activity of MexB. Recently, the crystal structure of MexB from $P$. aeruginosa was resolved and suggests a mechanism for substrate transport by MexB [24]. The residues at positions 136 in MexB are located in between the PN1 subdomain and the PN2 subdomain [24]. The residues at positions 681 in MexB are located in the PC2 subdomain [24]. The PC2 domain plays an important role in the formation of the entrance channel [24]. These data support the suggestion that Phe136 in MexB plays an important role in substrate extrusion by MexB.

MexAB-OprM inhibition by ABI showed that the LasR activation by 3-oxo-C9-HSL or 3-oxo-C10-HSL was similar to that in the mexB deletion mutant (Figures 1 and 3). The effect of ABI concentration on the response to 3-oxo-C12-HSL was lower than that of 3-oxo-C9HSL or 3-oxo-C10-HSL (Figure 3). These data suggest that the difference in the efflux ratio of 3-oxo-acyl-HSLs via MexAB-OprM may be due to differences in the acylside chain lengths; these differences in the efflux ratio were important in the response to the cognate 3-oxoC12-HSL in P. aeruginosa. However, we have to consider the degradation of acyl-HSLs by QS quenching lactonases or acylases, as well as LasR acyl-HSL binding activity in the acyl-HSLs response in $P$. aeruginosa. Previous studies showed that the substrate specificity of

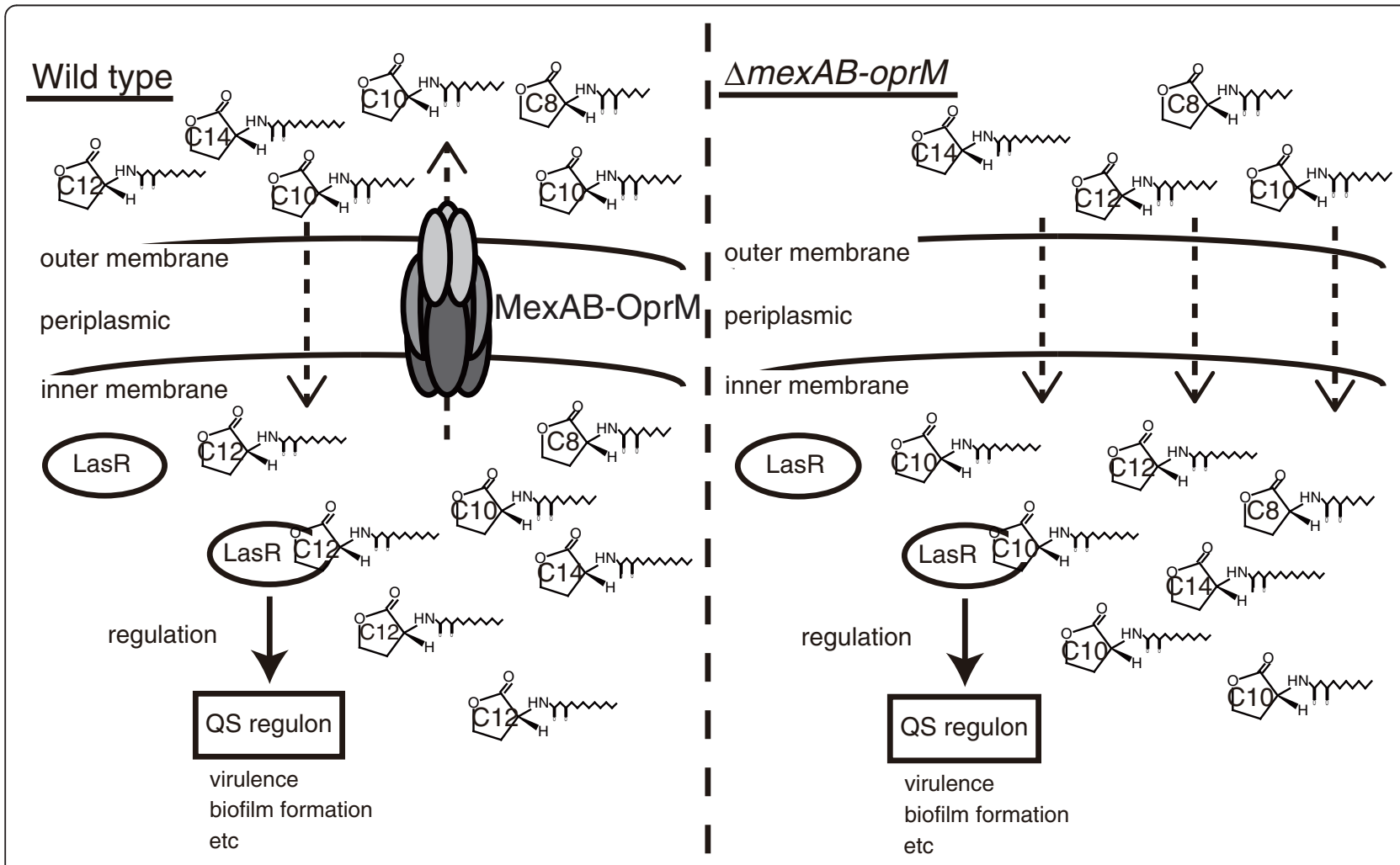

Figure 6 A model for QS regulation mechanism via the RND-type efflux pump MexAB-OprM. (a) MexAB-OprM extrudes 3-OxO-Cn-HSLS and controls the accessibility of non-cognate acyl-HSLs to LasR in P. aeruginosa QS-regulation. (b) In the P. aeruginosa MexAB-OprM mutant, noncognate 3-oxo-Cn-HSLs activate LasR. Non-cognate 3-oxo-Cn-HSLs-LasR complexes induce the wrong QS regulation. 
QS quenching enzymes was broad [25,26]. In addition, we showed the LasR responds to several acyl-HSLs by using the patulin competition assay (Figure 4). These results support the hypothesis that $P$. aeruginosa needs to use the acyl-HSLs selection system of MexAB-OprM in order to respond to cognate acyl-HSLs in mixed bacterial culture conditions. Furthermore, it is known that the concentrations of acyl-HSLs are high at high cell densities and LasR binds its specific acyl-HSL to activate the LasR regulon [4]. It was also suggested that MexABOprM regulates the concentration of acyl-HSLs in the cell via acyl-HSLs extrusion. The regulation of acylHSLs concentration via MexAB-OprM may therefore be important in the $P$. aeruginosa QS response.

The $P$. aeruginosa mexAB-oprM deletion mutant responded to 3-oxo-C10-HSL produced by $V$. anguillarum during $P$. aeruginosa-V. anguillarum co-cultivation (Figure 5). These results indicate that intracellular acylHSLs exported by MexAB-OprM regulated QS in $P$. aeruginosa. It has also been reported that the RND-type efflux pump BpeAB-OprB in B. pseudomallei is closely involved in bacterial communication [27,28]. These findings suggest that RND-type efflux pumps have a common ability for several acyl-HSL efflux systems. This selection mechanism may result in improved survival in mixed culture conditions.

\section{Conclusions}

This work demonstrates that MexAB-OprM does not control the binding of LasR to 3-oxo-Cn-HSLs but rather the accessibility of non-cognate acyl-HSLs to LasR in $P$. aeruginosa (Figure 6). Furthermore, the results indicate that QS is regulated by MexAB-OprM (Figure 6). MexAB-OprM not only influences multidrug resistance, but also selects acyl-HSLs and regulates QS in P. aeruginosa. The results demonstrate a new QS regulation mechanism via the efflux system MexAB-OprM in $P$. aeruginosa.

\section{Methods}

Bacterial strains, plasmids and growth conditions

The bacterial strains and plasmids used in this study are listed in Table 1. Bacterial cells were grown in LB broth or on $\mathrm{LB}$ agar at $37^{\circ} \mathrm{C}$ or $30^{\circ} \mathrm{C}$. The following antibiotics were added to media at the indicated concentrations: ampicillin, $100 \mu \mathrm{g} / \mathrm{ml}$ for E. coli; carbenicillin, $200 \mu \mathrm{g} / \mathrm{ml}$ for $P$. aeruginosa; tetracycline, $25 \mu \mathrm{g} / \mathrm{ml}$ for $E$. coli, $100 \mu \mathrm{g} / \mathrm{ml}$ for $P$. aeruginosa.

\section{Construction of knockout mutants lacking quorum sensing and efflux protein genes}

The $P$. aeruginosa mutants, KG7004 and KG7050, lacking quorum sensing and efflux protein genes were constructed by allele exchange using the plasmids listed in
Table 1, as described previously [30,35,42]. Construction of $P$. aeruginosa mutants in this study followed the order: PAO1 to KG7001 with plasI (for deletion of lasI), KG7001 to KG7004 with pAF2071 (for deletion of rhlI), and KG7004 to KG7050 with pMexB (for deletion of mex $B)$, respectively.

\section{Construction of QS reporter strains}

pSQG was constructed by subcloning a 700-bp EcoRI digested fragment derived from pGreen into the KpnI site of mini-CTX1 [38,39]. A lasB promoter- $g f p$ translational fusion was constructed by ligating a 591-bp fragment including the region encoding $\mathrm{N}$-terminal ten amino acids of LasB derived from the $P$. aeruginosa PAO1 chromosome. The resulting plasmid, pSQG003, was mobilized into KG7004 and KG7050 via E. coli S171. To accomplish excision, pFLP2, encoding Flp recombinase, was introduced into the $P$. aeruginosa KG7403 and KG7503 strains containing the las $B$ promoter-gfp translational fusion constructs by using the high transformation method and previously described procedures $[40,43]$.

In addition, the multicopy reporter plasmid pMQG003 was constructed. A lasB promoter-gfp translational fusion fragment from pSQG003 was cloned into pME6012 [41]. The lasB promoter- $g f p$ translational fusion fragment was prepared by using PCR with the primers CTX1-F (5' -CGATAGATCTGCCGTCCTTGCTGAAT TAGC-3') and CTX1-R (5'-AACTAGATCTCGCTTTT GAAGCTGATGTGC-3') containing an engineered restriction site BglII (forward and reverse). This fragment was restricted with $B g l \mathrm{II}$, and then ligated to the BglII site of pME6012.

\section{Construction of the plasmids expressing the wild-type and mutant mex $B$ genes in $P$. aeruginosa}

The stable $E$. coli-P. aeruginosa shuttle vector pKTA113 carrying mex $B$ was constructed in three steps. The first mex $B$ fragment amplified by PCR using the chromosomal DNA of $P$. aeruginosa PAO1 as a template and a pair of primers containing the engineered restriction sites HindIII ( $5^{\prime}$ ACATAAGCTTATGTCGAAGTTTTTCATTGATAGG -3') and SalI (5'- GCAATCTAGATTGCCCCTTTTCGACG GACG -3'). Next, mexB fragments were ligated to the multicloning site of pUC18 to yield pYT06. To obtain the MexB expression plasmid, a 3138-bp HindIII-XbaI fragment from pYT06 was ligated to the large HindIII-XbaI fragment of pTO003. The resulting construct containing MexB-6His under the lac promoter shall be referred to as pKTA113 in this paper.

To produce $\operatorname{mexB}$ mutants, the Stratagene Quickchange site-directed mutagenesis kit (Stratagene) was used according to the manufacturer's protocol. The Phe136Ala or Asp681Ala substitution was introduced 


\begin{tabular}{|c|c|c|}
\hline Strains/Plasmids & Characteristics & Reference \\
\hline \multicolumn{3}{|l|}{ Strains } \\
\hline \multicolumn{3}{|l|}{ P. aeruginosa } \\
\hline PAO1 & ATCC15692 & [29] \\
\hline KG4509 & $\Delta$ mexB derivative of $\mathrm{PAO} 1$ & This study \\
\hline KG7004 & $\Delta$ lasl $\Delta r h l l$ derivative of $\mathrm{PAO} 1$ & This study \\
\hline KG7050 & $\Delta$ lasl $\Delta r h l l \Delta m e x B$ derivative of $\mathrm{PAO} 1$ & This study \\
\hline KG7403 & gfp fused to the lasB promoter and integrated at the attB site of the KG7004 chromosome & This study \\
\hline KG7503 & gfp fused to the lasB promoter and integrated at the attB site of the KG7050 chromosome & This study \\
\hline \multicolumn{3}{|l|}{ E. coli } \\
\hline $\mathrm{DH} 5 \mathrm{a}$ & $\begin{array}{l}\mathrm{F}^{-} \text {, Ф80d lacZ } \triangle \mathrm{M} 15, \Delta(\operatorname{lac} Z Y A-\arg F) \cup 169 \text {, deoR, recA1, endA1, hsdR17 }\left(r_{\mathrm{k}}^{-} \mathrm{m}_{\mathrm{k}}^{+}\right), \text {phoA, supE44, } \\
\lambda^{-} \text {, thi-1, gyrA96, relA1 }\end{array}$ & {$[30]$} \\
\hline S17-1 & RE42-Tc: Mu-Km:: Tn7 pro res mod4 & [31] \\
\hline \multicolumn{3}{|l|}{ Plasmids } \\
\hline pUC18 & $A p^{r}$; high-copy-number cloning vector & [32] \\
\hline pBR322 & $A p^{r} T c^{r} ;$ high-copy-number cloning vector & [33] \\
\hline pSL1180 & super-polylinker phagemid & [34] \\
\hline pTO003 & $\mathrm{Gm}^{r}$; E. coli-P. aeruginosa shuttle expression vector & [35] \\
\hline pMT5059 & $\mathrm{Cb}^{\mathrm{r}} ; \mathrm{pBend} 2$ derivative carrying multiple-cloning site and Not I site & [36] \\
\hline pMT5071 & $\mathrm{Cm}^{r}$; pMOB3 derivative carrying $\Omega-\mathrm{Cm}$ instead of $\mathrm{Cm}$ & [37] \\
\hline pAF2071 & $\begin{array}{l}\mathrm{Cb}^{r} \mathrm{Cm}^{\mathrm{r}} \text {; pKT5059 carrying 2911-bp fragment with } 3^{\prime} \text { flanking region } \\
\text { of rhll including 91-bp of rhll and 2110-bp fragment with 5' flanking } \\
\text { region of rhll Mob cassette from pMT5071 at Not I }\end{array}$ & This study \\
\hline plasl & $\begin{array}{l}\mathrm{Cb}^{r} \mathrm{Cm}^{\mathrm{r}} \text {; pMT5059 carrying 1.0-kb PCR fragments with } 3^{\prime} \text { and } 5^{\prime} \text { flanking } \\
\text { regions of lasl and Mob cassette from pMT5071 at Not I }\end{array}$ & This study \\
\hline $\mathrm{pMexB}$ & $\begin{array}{l}\mathrm{Cb}^{\mathrm{r}} \mathrm{Cm}^{\mathrm{r}} ; \text { pMT5059 carrying 1.0-kb PCR fragments with } 3^{\prime} \text { and } 5^{\prime} \text { flanking } \\
\text { regions of mexB and Mob cassette from pMT5071 at Not I }\end{array}$ & This study \\
\hline pKTA113 & $\mathrm{Gm}^{\mathrm{r}}$; subcloning of full length mexB in Xba I and Hin dlll site of pTO003 & This study \\
\hline pYT57 & Phe136Ala mutation of mexB on pKTA113 & This study \\
\hline pYT81 & Asp681Ala mutation of mexB on pKTA113 & This study \\
\hline pGreen & Ap $^{r}$; Enhanced GFP cassette plasmid & [38] \\
\hline $\operatorname{mini}-\mathrm{CTX}_{1}$ & $\mathrm{TC}^{\mathrm{r}}$; self-profcient integration vector with tet, V-FRT-attP-MCS, ori, int, and oriT & [39] \\
\hline pSG & $T c^{r}$; subcloning of gfp from pGreen & This study \\
\hline pSQG003 & $\mathrm{TC}^{r}$; subcloning of a 598-bp lasB promoter region between Hin dIII and Kpn I sites of pSG & This study \\
\hline pFLP2 & $A p^{r}$; source of Flp recombinase & [40] \\
\hline pME6012 & Tcr; pVS1-p15A shuttle vector & [41] \\
\hline pMQG003 & Tc'; subcloning of a 1781-bp lasB promoter::gfp region from pSQG003 in Bg/ II site of pME6012 & This study \\
\hline
\end{tabular}

into pYT06, respectively. Then the mutated mexB fragments of the pYT06 mutants were subcloned into pTO003.

\section{Detection of lasB promoter activity by using GFP fluorescence intensity}

Cells were grown overnight at $30^{\circ} \mathrm{C}$ in $\mathrm{LB}$ medium with shaking. Overnight cultures were subcultured into fresh LB medium at a ratio of 1:100, grown under the same conditions for three hours, and then supplemented with
$5 \mu \mathrm{M}$ 3-oxo-Cn-HSL, respectively. Following an $8 \mathrm{~h}$ incubation at $30^{\circ} \mathrm{C}$, cells grown in LB with various acyl-HSLs were harvested by centrifugation, resuspended in phosphate-buffered saline, and then diluted with $200 \mu \mathrm{l}$ of phosphate-buffered saline. Green fluorescence of the reporter strains was measured using a Varioskan ${ }^{\text {TM }}$ microtiter plate reader (Thermo Fisher Scientific), with an excitation wavelength of $490 \mathrm{~nm}$ and emission detection at $510 \mathrm{~nm}$. Data are means \pm standard deviations for three independent experiments. The LasR inhibitor, Patulin was 
obtained from Wako-Pure Chemicals Ltd. (Osaka, Japan) [8]. The MexAB-OprM specific inhibitor, ABI ([[2(\{[((3R)-1-\{8-\{[(4-tert-butyl-1,3-thiazol-2-yl) amino $]$ carbonyl\}-4-oxo-3-[(E)-2-(1 H-tetrazol-5-yl)vinyl]-4 $H$-pyrido [1,2-a]pyrimidin-2-yl\} piperidin-3-yl)oxy]carbonyl 3amino) ethyl](dimethyl)ammonio]acetate, $\quad \mathrm{C}_{31} \mathrm{H}_{39} \mathrm{~N}_{11} \mathrm{O}_{6} \mathrm{~S} \cdot 6 \mathrm{H}_{2} \mathrm{O}$ ) was obtained from Daiichi Pharmaceutical Co., Ltd. (Tokyo, Japan) [44].

\section{Elastase assay by using FRET-AGLA}

The elastase activity in a $P$. aeruginosa culture supernatant was determined by using FRET-AGLA (see Additional file 3). Cells were grown under the same conditions as the lasB reporter assay. Cells grown in LB with various acyl-HSLs were harvested by centrifugation, and culture supernatants were recovered and filtered (0.22 $\mu \mathrm{m}$ pore-size filter). $50 \mu \mathrm{l}$ samples diluted 50 -fold were added to tubes containing $100 \mu \mathrm{l}$ of a FRET-AGLA solution (50 mM Tris- $\mathrm{HCl}, 200 \mathrm{mM} \mathrm{NaCl}(\mathrm{pH} 7.5)$, $10 \mathrm{mM} \mathrm{CaCl}_{2}, 0.4 \mathrm{mM}$ FRET-AGLA). The tubes were incubated for $15 \mathrm{~min}$ at $30^{\circ} \mathrm{C}$ and then $50 \mu \mathrm{l}$ of $1 \mathrm{M}$ $\mathrm{NaOH}$ was added. The degradation products of FRETAGLA produced by elastase were measured using the Varioskan ${ }^{\mathrm{TM}}$ microtiter plate reader with an excitation wavelength of $355 \mathrm{~nm}$ and emission detection at $460 \mathrm{~nm}$. The resolution rate of the degradation products of FRET-AGLA was determined by extrapolating the obtained fluorescence of the degradation products of FRET-AGLA on a standard curve.

\section{Cross-streaking experiments}

The monitor strains, KG7004(pMQG003) or KG7050 (pMQG003), and the respective test strains were streaked close to each other on nutrient agar plates (Nissui, Tokyo, Japan) (see Additional file 3). Following $24 \mathrm{~h}$ incubation at $30^{\circ} \mathrm{C}$, the plates were illuminated with blue light using an SZX-FGFP filter in combination with a halogen lamp as a light source, and green fluorescence was observed under a Stereomicroscope SZX12 system (Olympus).

\section{Additional files}

Additional file 1: Figure S1. Cross-streak experiment for detection of bacterial interaction via acyl-HSLs. The two monitor strains used

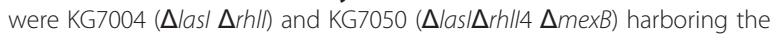
lasB promoter-afp plasmid (pMQG003) were used. Test strains against the monitor strains (center) were cross-streaked on LB agar plates. Following $24 \mathrm{~h}$ incubation at $30^{\circ} \mathrm{C}$, the growth of strains was observed under a stereomicroscope, and then production of GFP by the monitor strains was visualized by excitation of the plates with blue light.

Additional file 2: Figure S2. TLC analysis of 3-oxo-C10-HSL produced by $V$. anguillarum. Extracted samples from $V$. anguillarum cultures were chromatographed on a C-18 RP-TLC plate, developed with methanol/water $(70: 30, v / v)$. The spots were visualized 13 by overlaying the TLC plate with C. violaceum VIR07. As AHL standards, Cn-HSL: 14 C6-
HSL, C8-HSL and C10-HSL, 3-oxo-Cn-HSL: 3-oxo-C6-HSL, 3-oxo-C8-HSL, 15 3-oxo-C10-HSL and 3-oxo-C12-HSL were used.

Additional file 3: Supplemental information of Materials, Methods, Figure legend of Figure S1 and S2 and References [1,45-49].

\section{Authors' contributions}

SM, and SS carried out the elastase assay and lasB reporter assay. HI carried out cross-streak experiments. TK constructed lasB promoter-gfp reporter strains. SM synthesized FRET-AGLA, elastase substrate. MH synthesized acylHSLS. JO and NG conceived of the study, and participated in its design and coordination and helped to draft the manuscript. All authors read and approved the final manuscript.

\section{Acknowledgements}

We thank Herbert P. Schweizer (Colorado State University, USA) and the National Institute of Genetics (Mishima, Japan) for providing mini-CTX1 and pGreen, respectively. This research was supported by Grant-in-Aids for Young Scientists (B) to S. Minagawa, and for Scientific Research (C) to N. Gotoh and S. Miyairi, from Japan Society for the Promotion of Science (JSPS), and a grant from the Institute for Fermentation, Osaka to N. Gotoh.

\section{Author details}

'Department of Microbiology and Infection Control Science, Kyoto Pharmaceutical University, Yamashina, Kyoto 607-8414, Japan. 'Laboratory of Organic Chemistry, School of Pharmacy, Nihon University, Funahashi, Chiba 274-8555, Japan. ${ }^{3}$ Suntory Foundation for Life Science, Bioorganic Research Institute, Mishima, Osaka 618-8503, Japan.

Received: 2 December 2011 Accepted: 13 April 2012

Published: 10 May 2012

\section{References}

1. Fuqua C, Greenberg EP: Listening in on bacteria: acyl-homoserine lactone signaling. Nat Rev 2002, 3:685-695.

2. Waters CM, Bassler BL: Quorum sensing: cell-to-cell communication in bacteria. Annu Rev Cell Dev Biol 2005, 21:319-346.

3. Duan K, Surcttc MG: Environmental regulation of Pseudomonas aeruginosa PAO1 Las and Rhl quorum-sensing system. J Bacteriol 2007, 189:4827-4836.

4. Schuster M, Lostroh CP, Ogi T, Greenberg EP: Identification, timing, and signal specificity of Pseudomonas aeruginosa quorum-controlled genes: a transcriptome analysis. J Bacteriol 2003, 185:2066-2079.

5. Wagner VE, Li LL, Isabella VM, Iglewski BH: Analysis of the hierarchy of quorum-sensing regulation in Pseudomonas aeruginosa. Anal Bioanal Chem 2007, 387:469-479.

6. Bottomley MJ, Muraglia E, Bazzo R, Carfi A: Molecular insights into quorum sensing in the human pathogen Pseudomonas aeruginosa from the structure of the virulence regulator LasR bound to its autoinducer. J Biol Chem 2007, 282:13592-13600.

7. Dubem JF, Diggle SP: Quorum sensing by 2-alkyl-4-quinolones in Pseudomonas aeruginosa and other bacterial species. Mol Biosyst 2008, 4:882-888.

8. Pearson JP, Gray KM, Passador L, Tucker KD, Eberhard A, Iglewski BH, Greenberg EP: Structure of the autoinducer required for expression of Pseudomonas aeruginosa virulence genes. Proc Natl Acad Sci USA 1994, 91:197-201.

9. Bredenbruch F, Geffers R, Nimlz M, Buer J, Haussler S: The Pseudomonas aeruginosa quinolone signal (PQS) has an iron-chelating activity. Environ Microbiol 2006, 8:1318-1329.

10. Vannini A, Volpari C, Gargioli C, Muraglia E, Cortese R, Francesco RD, Neddermann P, Marco SD: The crystal structure of the quorum sensing protein TraR bound to its autoinducer and target DNA. EMBO J 2002, 31:4393-4401

11. Riedel K, Hentzer M, Geisenberger O, Huber B, Steidle A, Wu H, Hoiby N, Givskov M, Molin S, Eberl L: N-Acylhomoserine-lactone-mediated communication between Pseudomonas aeruginosa and Burkholderia cepacia in mixed biofilms. Microbiology 2001, 147:3249-3262.

12. Piddock $\sqcup$ : Multidrug-resistance efflux pumps - not just for resistance. Nat Rev Microbiol 2006, 4:629-636. 
13. Evans K, Passador L, Srikumar R, Tsang E, Nezezon J, Poole K: Influence of the MexAB-OprM multidrug efflux system on quorum sensing in Pseudomonas aeruginosa. J Bacteriol 1998, 180:5443-5447.

14. Pearson JP, Delden CV, Iglewski BH: Active efflux and diffusion are involved in transport of Pseudomonas aeruginosa cell-to-cell signals. J Bacteriol 1999, 181:1203-1210.

15. Hirakata Y, Srikumar R, Poole K, Gotoh N, Suematsu T, Kohno S, Kamihira S, Hancock RTW, Speert DP: Multidrug efflux systems play an important role in the invasiveness of Pseudomonas aeruginosa. J Exp Med 2002, 196:109-118.

16. Masuda N, Sakagawa E, Ohya S, Gotoh N, Tsujimoto H, Nishino T: Substrate specificities of MexAB-OprM, MexCD-OprJ, and MexXY-OprM efflux pumps in Pseudomonas aeruginosa. Antimicrob Agents Chemother 2000, 44:3322-3327

17. Murakami S, Nakashima R, Yamashita E, Yamaguchi A: Crystal structure of bacterial multidrug efflux transporter AcrB. Nature 2002, 419:587-593.

18. Murakami S, Nakashima R, Yamashita E, Matsumoto T, Yamaguchi A: Crystal structures of a multidrug transporter reveal a functionally rotating mechanism. Nature 2006, 443:173-179.

19. Zhu J, Chai Y, Zhong Z, Li S, Winans SC: Agrobacterium bioassay strain for ultrasensitive detection of $\mathrm{N}$-acylhomoserine lactone-type quorumsensing molecules: Detection of autoinducers in Mesorhizobium huakuii. Appl Environ Microbiol 2003, 69:6949-6953.

20. Milton DL, Chalker VJ, Kirke DK, Hardman A, Mara MC, Williams P: The LuxM homologue VanM from Vibrio anguillarum directs the synthesis of $\mathrm{N}$-(3hydroxyhexanoyl) homoserine Lactone and $\mathrm{N}$-hexanoylhomoserine lactone. J Bacteriol 2001, 183:3537-3547.

21. Swift S, Winson MK, Chan PF, Bainton NJ, Birdsall M, Reeves PJ, Rees CED Chhabra SR, Hill PJ, Throup JP, Bycroft BW, Salmond GPC, Williams P, Stewart GSAB: A novel strategy for the isolation of luxl homologues: evidence for the widespread distribution of a LuxR: Luxl superfamily in enteric bacteria. Mol Microbiol 2006, 10:511-520.

22. Morohoshi T, Kato M, Fukamachi K, Kato N, Ikeda T: N-Acylhomoserine lactone regulates violacein production in Chromobacterium violaceum type strain ATCC12472. FEMS Microbiol Lett 2008, 279:124-130.

23. Shaw PD, Ping G, Daly SL, Cha C, Cronan JE Jr, Rinehart KL, Farrand SK: Detecting and characterizing $\mathrm{N}$-acyl-homoserine lactone signal molecules by thin-layer chromatography. Proc Natl Acad Sci USA 1997, 94:6036-6041.

24. Sennhauser G, Bukowska MA, Briand C, Gru"tter MG: Crystal structure of the multidrug exporter MexB from Pseudomonas aeruginosa. J Mol Biol 2009, 389:134-145.

25. Wang $L H$, Weng $L X$, Dong $Y H$, Zhang $L H$ : Specificity and enzyme kinetics of the quorum-quenching $\mathrm{N}$-Acyl homoserine lactone lactonase $(\mathrm{AHL}$ lactonase). J Biol Chem 2004, 279:13645-13651.

26. Sio CF, Otten LG, Cool RH, Diggle SP, Braun PG, Bos R, Daykin M, Cámara M, Williams P, Quax WJ: Quorum quenching by an N-acyl-homoserine lactone acylase from Pseudomonas aeruginosa PAO1. Infect Immun 2006, 74:1673-1682.

27. Chan YY, Bian HS, Tan TMC, Mattmann ME, Geske GD, Igarashi J, Hatano T, Suga $\mathrm{H}$, Blackwell HE, Chua $\mathrm{KL}$ : Control of quorum sensing by a Burkholderia pseudomallei multidrug efflux pump. J Bacteriol 2007, 189:4320-4324.

28. Chan YY, Chua K: The Burkholderia pseudomallei BpeAB-OprB efflux pump: Expression and impact on quorum sensing and virulence. J Bacteriol 2005, 187:4707-4719.

29. Stover CK, Pham XQ, Erwin AL, Mizoguchi SD, Warrener P, Hickey MJ, Brinkman FS, Hufnagle WO, Kowalik DJ, Lagrou M, Garber RL, Goltry L, Tolentino E, Westbrock-Wadman S, Yuan Y, Brody LL, Coulter SN, Folger KR, Kas A, Larbig K, Lim R, Smith K, Spencer D, Wong GK, Wu Z, Paulsen IT, Reizer J, Saier MH, Hancock RE, Lory S, Olson MV: Complete genome sequence of Pseudomonas aeruginosa PAO1, an opportunistic pathogen. Nature 2000, 406:959-964.

30. Sambrook J, Fritsch EF, Maniatis TA: Molecular Cloning: A Laboratory Manual. 2nd edition. NY, U.S.A: Cold Spring Harbor; 1989.

31. Simon R, Priefer U, Plihler A: A broad host range mobilization system for in vivo genetic engineering: transposon mutagenesis in gram-negative bacteria. Biotechnology 1983, 1:784-794.

32. Yanisch-Perron C, Vieira J, Messing J: Improved M13 phage cloning vectors and host strains: nucleotide sequences of the M13mpl8 and pUC19 vectors. Gene 1985, 33:103-119.
33. Sutcliffe JG: Complete nucleotide sequence of the Escherichia coli plasmid pBR322. Cold Spring Harb. Symp. Quant. Biol 1979, 43:77-90.

34. Brosius J: Superpolylinkers in cloning and expression vectors. DNA 1989, 8:759-777

35. Murata T, Kuwagaki M, Shin T, Gotoh N, Nishino T: The substrate specificity of tripartite efflux systems of Pseudomonas aeruginosa is determined by the RND component. Biochem Biophys Res Commun 2002, 299:247-251.

36. Tsuda M, Miyazaki H, Nakazawa T: Genetic and physical mapping of genes involved in pyoverdine production in Pseudomonas aeruginosa PAO1. J Bacteriol 1995, 177:423-431.

37. Tsuda M: Use of a transposon-encoded site-specific resolution system for construction of large and defined deletion mutations in bacterial chromosome. Gene 1998, 207:33-42.

38. Miller WG, Lindow SE: An improved GFP cloning cassette designed for prokaryotic transcriptional fusions. Gene 1997, 191:149-153.

39. Hoang TT, Kutchma AJ, Becher A, Schweizer HP: Integration-proficient plasmids for Pseudomonas aeruginosa: Site-specific integration and use for engineering of reporter and expression strains. Plasmid 2000, 43:59-72.

40. Hoang TT, Karkoff-Schweizer RR, Kutchma AJ, Schweizer HP: A broad-hostrange Flp-FRT recombination system for site-specific excision of chromosomally-located DNA sequences: application for isolation of unmarked Pseudomonas aeruginosa mutants. Gene 1998, 212:77-86.

41. Heeb S, Itoh Y, Nishijyo T, Schnider U, Keel C, Wada J, Walsh U, O' Gara F, Haas D: Small, stable shuttle vectors based on the minimal pVS1 replicon for use in gram-negative, plant-associated bacteria. Mol Plant Microbe Interact 2000, 13:232-237.

42. Murata T, Gotoh N, Nishino T: Characterization of outer membrane efflux proteins OpmE, OpmD and OpmB of Pseudomonas aeruginosa: molecular cloning and development of specific antisera. FEMS Microbiol Lett 2002, 217:57-63.

43. Choi KH, Kumar A, Schweizer HP: A 10-min method for preparation of highly electrocompetent Pseudomonas aeruginosa cells: Application for DNA fragment transfer between chromosomes and plasmid transformation. J Microbiol Methods 2006, 64:391-397.

44. Yoshida K, Nakayama K, Ohtsuka M, Kuro N, Yokomizo Y, Sakamoto A, Takemura M, Hoshino K, Kanda H, Nitanai H, Namba K, Yoshida K, Imamura Y, Zhang JZ, Lee VJ, Watkins WJ: MexAB-OprM specific efflux pump inhibitors in Pseudomonas aeruginosa. Part 7: Highly soluble and in vivo active quaternary ammonium analogue D13-9001, a potential preclinical candidate. Bioorg Med Chem 2007, 15:7087-7097.

45. Horikawa M, Tateda K, Tuzuki E, Ishii Y, Ueda C, Takabatake T, Miyairi S, Yamaguchi K, Ishiguro M: Synthesis of Pseudomonasquorum-sensing autoinducer analogs and structural entities required for induction of apoptosis in macrophages. Bioorg. Med. Chem. Lett 2006, 16:2130-2131.

46. Nishino N, Powers JC: Pseudomonas aeruginosaelastase: Development of a new substrate, inhibitors, and an affinity ligand. J Biol Chem 1980, 255:3482-3486

47. Chin-A-Woeng TF, van den Broek D, de Voer G, van der Drift KM, Tuinman S, Thomas-Oates JE, Lugtenberg BJ, Bloemberg GV: Phenazine-1carboxamide production in the biocontrol strain Pseudomonas chlororaphisPCL1391 is regulated by multiple factors secreted into the Growth Medium. Mol Plant Microbe Interact 2001, 14:969-979.

48. Laue BE, Jiang Y, Chhabra SR, Jacob S, Stewart GSAB, Hardman A, Downie JA, O' Gara F, Williams P: The biocontrol strain Pseudomonas fluorescensF113 produces the Rhizobium small bacteriocin, $\mathrm{N}$-(3-hydroxy7-cis-tetradecenoyl) homoserine lactone, via HdtS, a putative novel $\mathrm{N}$ acylhomoserine lactone synthase. Microbio/ 2000, 146:2469-2480.

49. Holden MT, Ram Chhabra S, de Nys R, Stead P, Bainton NJ, Hill PJ, Manefield M, Kumar N, Labatte M, England D, Rice S, Givskov M, Salmond GP, Stewart GS, Bycroft BW, Kjelleberg S, Williams P: Quorum-sensing cross talk: isolation and chemical characterization of cyclic dipeptides from Pseudomonas aeruginosa and other gram-negative bacteria. Mol Microbiol 1999, 33:1254-1266.

doi:10.1186/1471-2180-12-70

Cite this article as: Minagawa et al.: RND type efflux pump system MexAB-OprM of pseudomonas aeruginosa selects bacterial languages, 3oxo-acyl-homoserine lactones, for cell-to-cell communication. BMC Microbiology 2012 12:70. 